\title{
Correction to: EcoDesign and Sustainability II
}

\author{
Yusuke Kishita, Mitsutaka Matsumoto, Masato Inoue, \\ and Shinichi Fukushige
}

\section{Correction to:}

Y. Kishita et al. (eds.), EcoDesign and Sustainability II, Sustainable Production, Life Cycle Engineering and Management, https://doi.org/10.1007/978-981-15-6775-9

In the original version of the book, the following belated corrections have been incorporated:

In chapter "Embedding a Sustainability Focus in Packaging Development Processes", the author group was incorrect. The author group "Kenichiro Chinen, Hideki Endo, Mitsutaka Matsumoto, and Yongliang Stanley Han" has been changed to "Bjorn de Koeijer, Iris Borgman, Jörg Henseler, Roland ten Klooster, and Jos de Lange" in the Frontmatter, Backmatter and in the Chapter.

In chapter "Consumer's Perception of Plastics in Everyday Products in Relation to Their Personality", the author group was incorrect. The author group "Kenichiro Chinen, Hideki Endo, Mitsutaka Matsumoto, and Yongliang Stanley Han" has been changed to "Lore Veelaert, Els Du Bois, Laure Herweyers, and Ingrid Moons" in the Frontmatter, Backmatter and in the Chapter.

This has been corrected in the updated version.

The updated version of these chapters can be found at https://doi.org/10.1007/978-981-15-6775-9_4

https://doi.org/10.1007/978-981-15-6775-9_5 